\title{
PROBLEMAS DE ESCRITURA IDENTIFICADOS EN LA PRODUCCIÓN DE ENSAYOS DE ESTUDIANTES DE TRES CARRERAS DE PEDAGOGÍA
}

\section{WRITING PROBLEMS IDENTIFIED IN THE PRODUCTION OF STUDENTS' ESSAYS IN THREE TEACHING PROGRAMS}

Paulina Meza*

\begin{abstract}
RESUMEN: El objetivo general de esta investigación es determinar la variación de los problemas de escritura identificados en ensayos argumentativos producidos por estudiantes de primer año de tres carreras de pedagogía de una universidad chilena. Para ello, en el marco de una investigación cualitativa y un diseño no experimental, analizamos un corpus constituido por 12 ensayos argumentativos. Para analizar este corpus utilizamos una rúbrica analítica que fue validada por distintos especialistas. Entre los principales resultados destacamos que, en general, los estudiantes de Pedagogía en Castellano y Filosofía obtienen mejores resultados que los de Pedagogía en Educación Diferencial y de Pedagogía en Educación General Básica. Además, pudimos identificar las dimensiones que representan mayores dificultades para los estudiantes al momento de desarrollar un ensayo argumentativo. Ellas son: referencias bibliográficas, puntuación y cohesión en Pedagogía en Educación Diferencial y Pedagogía en Educación General Básica. Los ensayos de Pedagogía en Castellano y Filosofía, por su parte, no evidencian dimensiones que no alcancen el porcentaje mínimo de aprobación. Finalmente, nuestros resultados pueden servir como insumo para sustentar una didáctica de la escritura en carreras de formación de profesores, pues nuestros resultados podrían entregar pistas sobre los elementos del ensayo que deberían trabajarse con mayor dedicación en las carreras consideradas en esta investigación.
\end{abstract}

Palabras clave: Escritura; Género ensayo; Pedagogía; Formación de profesores; Problemas de producción de textos.

ABSTRACT: The general aim is to determine the variation of the writing problems identified in argumentative essays produced by first-year students from three teaching programs at a Chilean university. Within the framework of a qualitative research and a non-experimental design, we analyzed a corpus consisting of 12 argumentative essays. To analyze this corpus, we used an analytical rubric that was

*Universidad de La Serena (Chile). 
validated by different specialists. Among the main results, we highlight that, in general, students of Pedagogy in Spanish and Philosophy obtain better results than those of Pedagogy in Differential Education and Pedagogy in Basic General Education. In addition, we were able to identify the dimensions that represent the greatest difficulties for students when developing an argumentative essay. They are bibliographic references, punctuation and cohesion in Pedagogy in Differential Education and Pedagogy in Basic General Education. On the other hand, the essays on Pedagogy in Spanish and Philosophy do not show dimensions that do not reach the minimum percentage of approval. Finally, our results can serve as an input to support a didactic of writing in teacher training careers, since our results could provide clues about the elements of the essay that should be worked with greater dedication in the careers considered in this research.

Keywords: Writing; Essay genre; Pedagogy; Teacher training; Text production problems.

\section{INTRODUCCIÓN}

Diversas investigaciones han evidenciado las dificultades y carencias que poseen los estudiantes universitarios para producir textos generales y académicos (PINETEH, 2014; HERMOSILLO y VERDÍN, 2019; MEZA, CASTELLÓN y GLADIC, 2021; entre muchos otros). De hecho, se ha afirmado que, en educación superior, la escritura es una de las habilidades más difíciles de desarrollar (NGUYEN, 2019). Probablemente, esto se debe a la diferencia que existe entre las prácticas de escritura que se utilizan en la educación secundaria y las habilidades que demanda la formación universitaria en el ámbito de la producción de textos (OYARZÚN y VALDÉS, 2019). Esta falta de dominio de las habilidades de escritura deriva en consecuencias sumamente indeseables como, por ejemplo, reprobación, retraso y deserción de los estudiantes (OLAVE-ARIAS, CISNEROS-ESTUPIÑÁN y ROJASGARCÍA, 2013; URIBE y CARRILLO, 2014). Con el propósito de entregarles mayor cantidad de herramientas para que puedan enfrentar más exitosamente la producción de textos en contextos académicos, en los últimos años, las instituciones de educación superior en Chile han integrado sistemática y transversalmente cursos 
de habilidades comunicativas en los niveles iniciales de las distintas carreras de pregrado.

En este marco, diversas investigaciones se han interesado por reconocer cuáles son los principales problemas que presentan los textos producidos por estudiantes durante su formación de pregrado. Así, contamos, por ejemplo, con investigaciones que han reportado los problemas que presentan los estudiantes en educación superior toda vez que se enfrentan a la escritura en una segunda lengua (KHUWAILEH y SHOUMALI, 2000; AL FADDA, 2012; HUANG y JUN ZHANG, 2020; NGUYEN, 2019). En el contexto chileno, se han reportado los errores más frecuentes que cometen los estudiantes en ámbitos disciplinares y géneros específicos como el Informe Académico en Enfermería (ARECHABALA et al., 2011) y los géneros profesionales con fines académicos en Derecho y Medicina (MEZA, CASTELLÓN y GLADIC, 2021).

Por otro lado, se han desarrollado investigaciones que se han enfocado en el género ensayo con el propósito de identificar las dificultades discursivas y personales de los estudiantes de una carrera de pedagogía (ERRÁZURIZ et al., 2015), de evaluar su proceso de escritura (ERRÁZURIZ, 2019) o de analizar el uso de los marcadores discursivos en dicho género (ERRÁZURIZ, 2012). Desde un punto de vista léxico, se han identificado expresiones formulaicas (JAWORSKA, KRUMMES y ENSSLIN, 2015) o paquetes léxicos en corpus de ensayos de estudiantes universitarios (SHIN, 2019).

A pesar de estos importantes avances, hasta la fecha, no encontramos investigaciones que hayan identificado los problemas de escritura que presentan los estudiantes de ingreso de distintas carreras de pedagogía en Chile. En este contexto, los objetivos de esta investigación son: a) identificar los problemas que dificultan la producción de ensayos argumentativos por parte de estudiantes de primer año de tres carreras de pedagogía de una universidad chilena; b) determinar la variación disciplinar de los problemas identificados en la producción de ensayos argumentativos por parte de estudiantes de primer año de tres carreras de 
pedagogía de una universidad chilena. Para ello, a partir de una investigación cualitativa, hemos analizado un corpus constituido por 12 ensayos argumentativos producidos por estudiantes de primer año de Pedagogía en Educación General Básica, Pedagogía en Educación Diferencial y Pedagogía en Castellano y Filosofía.

La investigación propuesta se justifica si consideramos, además del vacío declarado, el rol fundamental que posee el ensayo argumentativo en el contexto universitario (SHIN, 2019). Además, como afirma Shin (2019, p. 3), "more research on argumentative essay writing is needed, particularly because the basic function of this genre, which is to present and develop an argument, is a key skill across academic disciplines". Asimismo, esta investigación podría ser útil para guiar el proceso didáctico de producción de un ensayo argumentativo, pues, como señalan Errázuriz et al. (2015) siguiendo a Castro, Hernández y Sánchez(2010), a pesar de la constante presencia del ensayo en el ámbito universitario y en la escritura académica, aún no están bien definidas sus características, ni las prácticas metodológicas adecuadas para su enseñanza de acuerdo con cada ámbito, hechos que derivan en que los estudiantes presenten dificultades al momento de producir un ensayo. Por otro lado, los docentes no cuentan con las herramientas suficientes para guiar a sus estudiantes en la producción de un ensayo.

Como concepto teórico central de esta investigación destacamos el ensayo argumentativo. Al respecto, se ha señalado que el ensayo es el género que se produce más comúnmente durante la formación de pregrado (WINGATE, 2012). En la misma línea, se puede agregar que es el género que se exige en la mayoría de las universidades, especialmente, en el área de Humanidades (ERRÁZURIZ et al., 2015). La presencia constante del género en cuestión en esta área podría deberse a que "como ningún otro género, [el ensayo] sintetiza las formas típicas de pensar en el ámbito de las Humanidades (CASTRO et al., 2010, p. 55).

El ensayo argumentativo, particularmente, tiene como propósito comunicativo argumentar a favor de un determinado punto de vista. Por ello, escribir un ensayo argumentativo requiere pensamiento crítico y lógico, además de la 
capacidad de incorporar fuentes de manera coherente. Asimismo, comprende habilidades como la reflexión, el análisis, la crítica y la opinión (CASTRO et al., 2010; ERRÁZURIZ et al., 2015; PARKINSON y MUSGRAVE, 2014). Dado que el ensayo argumentativo se produce en contextos académicos, resulta esperable que contenga las características de la escritura académica como, por ejemplo, nominalizaciones, estructuras argumentativas extensas, análisis crítico (JAWORSKA et al., 2015). Asimismo, es esperable que se respeten las convenciones de algún sistema de normas de estilo de citas y referencias.

A continuación, presentamos el sustento metodológico de la investigación. Posteriormente, damos cuenta del análisis y discusión de resultados. Finalmente, damos cierre al trabajo con la exposición de las conclusiones.

\section{MARCO METODOLÓGICO}

La investigación presentada tiene dos objetivos centrales, a saber: a) identificar los problemas que dificultan la producción de ensayos argumentativos por parte de estudiantes de primer año de tres carreras de pedagogía de una universidad chilena; b) determinar la variación disciplinar de los problemas identificados en la producción de ensayos argumentativos por parte de estudiantes de primer año de tres carreras de pedagogía de una universidad chilena. Para cumplir con estos propósitos desarrollamos una investigación cualitativa basada en un diseño no experimental (HERNÁNDEZ et al., 2014; CRESWELL y CRESWELL, 2018).

En cuanto al corpus de análisis, los textos recogidos fueron producidos en el contexto de un curso orientado al desarrollo de las competencias de comunicación oral y escrita. Si bien este curso es transversal a todas las carreras de pedagogía de la Universidad de La Serena (Chile), cada carrera cursó la asignatura de forma separada, vale decir, se dictó tres veces el mismo curso (una vez por cada carrera). 
Más específicamente, el corpus analizado está constituido por 12 ensayos argumentativos producidos por estudiantes de primer año de tres carreras de formación de profesores de la Universidad de La Serena (Chile), distribuidos de la siguiente forma: 4 ensayos de Pedagogía en Educación General Básica, 4 de Pedagogía en Educación Diferencial y 4 de Pedagogía en Castellano y Filosofía. Cada ensayo tiene, en promedio, 1.000 palabras, por lo que el corpus alcanza un total de 12.477 palabras. Cabe señalar, además, que los textos fueron producidos durante el año 2020, por lo que, debido a la pandemia por COVID-19, las clases en las que se entregaron las herramientas para producir el ensayo fueron desarrolladas de forma telemática. Antes de entregar su ensayo para someterlo a evaluación, los estudiantes contaron con 8 sesiones en las que recibieron los contenidos teóricos y realizaron actividades prácticas en relación con la tarea de escritura solicitada. Durante este periodo, además, tuvieron la posibilidad de solicitar retroalimentación sobre el avance de sus trabajos a la profesora encargada del curso.

Por otro lado, en cuanto a los aspectos éticos de la investigación, es importante explicitar que los estudiantes facilitaron sus ensayos evaluados de forma voluntaria. Además, firmaron un consentimiento informado en el que declararon que facilitaban sus textos para esta investigación. Dada esta entrega voluntaria de los trabajos, pudimos acceder solo a un porcentaje de los ensayos producidos en cada curso.

Para evaluar los textos recogidos, se utilizó un instrumento diseñado especialmente con el propósito de evaluar ensayos argumentativos de estudiantes de primer año. Particularmente, se trata de una rúbrica analítica, que fue socializada con los estudiantes antes de comenzar con la escritura de su ensayo. A continuación, en la Tabla 1, se presentan las dimensiones que constituyen la rúbrica utilizada junto a una breve explicación del aspecto que se evalúa en cada una de ellas: 
Tabla 1- Descripción del instrumento de evaluación

\begin{tabular}{|c|c|}
\hline DIMENSIÓN & ASPECTO EVALUADO \\
\hline $\begin{array}{l}\text { 1. Situación } \\
\text { retórica }\end{array}$ & $\begin{array}{l}\text { El ensayo se adecua a los elementos de la situación } \\
\text { retórica establecidos: tema, audiencia y propósito. }\end{array}$ \\
\hline 2. Título & $\begin{array}{l}\text { El título es representativo del tópico tratado y atractivo } \\
\text { para el lector. }\end{array}$ \\
\hline 3. Introdi & $\begin{array}{l}\text { La introducción desarrolla adecuadamente sus } \\
\text { elementos retóricos constitutivos. }\end{array}$ \\
\hline 4. Desc & $\begin{array}{l}\text { Se presentan correctamente tres argumentos distintos } \\
\text { con su respectivo apoyo. }\end{array}$ \\
\hline 5. Cierre & $\begin{array}{l}\text { El cierre presenta adecuadamente al menos tres de sus } \\
\text { elementos retóricos constitutivos. }\end{array}$ \\
\hline $\begin{array}{l}\text { 6. Elementos para } \\
\begin{array}{l}\text { llamar } \\
\text { atención da } \\
\text { lector }\end{array}\end{array}$ & $\begin{array}{l}\text { Se incorpora adecuadamente al menos un elemento } \\
\text { que contribuye a llamar la atención del lector (anécdota, } \\
\text { cita, interrogante, analogía, etc.). }\end{array}$ \\
\hline 7. Cohe & $\begin{array}{l}\text { Se evidencia la utilización adecuada y variada de } \\
\text { mecanismos de cohesión para guiar la organización del } \\
\text { texto. }\end{array}$ \\
\hline $\begin{array}{l}\text { 8. Modos de } \\
\text { organización del } \\
\text { discurso }\end{array}$ & $\begin{array}{l}\text { Se evidencia el uso predominante del modo } \\
\text { argumentativo y los otros modos se utilizan como apoyo } \\
\text { a la argumentación. }\end{array}$ \\
\hline 9. Personalización & $\begin{array}{l}\text { Se evidencia un uso consistente y adecuado de una } \\
\text { persona gramatical. }\end{array}$ \\
\hline $\begin{array}{l}\text { 10. Referencias } \\
\text { bibliográficas }\end{array}$ & $\begin{array}{l}\text { Las citas y referencias bibliográficas se ajustan al } \\
\text { formato de APA } 7 .\end{array}$ \\
\hline 11. Ortografía & Existe un uso correcto de la ortografía acentual y literal. \\
\hline 12. Puntuación & Existe un uso correcto de la puntuación. \\
\hline
\end{tabular}




\begin{tabular}{c|l}
\hline $\begin{array}{c}\text { 13. Aspectos } \\
\text { formales }\end{array}$ & $\begin{array}{l}\text { El trabajo cumple con las especificaciones de formato } \\
\text { indicadas (tipo y tamaño de fuente, interlineado, etc.). }\end{array}$ \\
\hline
\end{tabular}

Fuente: Preparado por la autora

Cabe señalar que, como procedimiento de validación, el instrumento de evaluación fue socializado con dos docentes con experiencia en cursos de habilidades comunicativas y, además, con la ayudante de los cursos en los que se produjeron los ensayos analizados en esta investigación. Estas tres personas, que actuaron como jueces, validaron el instrumento desarrollado y entregaron algunas observaciones y sugerencias, las que fueron incorporadas en la versión final de la rúbrica.

Es importante agregar que, tras la aplicación de la rúbrica a los ensayos, para distinguir las dimensiones logradas de las no logradas, establecimos un umbral de $60 \%$ como nivel de logro. Ello nos permitió identificar los problemas más graves que tienen los estudiantes al momento de producir sus ensayos argumentativos. Como hemos señalado en una investigación anterior (MEZA, CASTELLÓN y GLADIC, 2021), la determinación de dicho umbral obedece a dos razones centrales, primero, debido a que el nivel de exigencia de aprobación para los estudiantes en las carreras de pedagogía incluidas en la investigación es de $60 \%$. Y, segundo, porque dicho umbral ha sido escogido en otras investigaciones en las que se estudian distintos géneros académicos para establecer el estatus de una categoría (IBÁÑEZ, 2008; KANOKSILAPATHAM, 2007; MARTÍNEZ 2015; MEZA y DA CUNHA, 2019).

Por último, en cuanto a los procedimientos de análisis, ellos se sintetizan, a continuación, en la Figura 1: 
Figura 1- Procedimientos de análisis del corpus

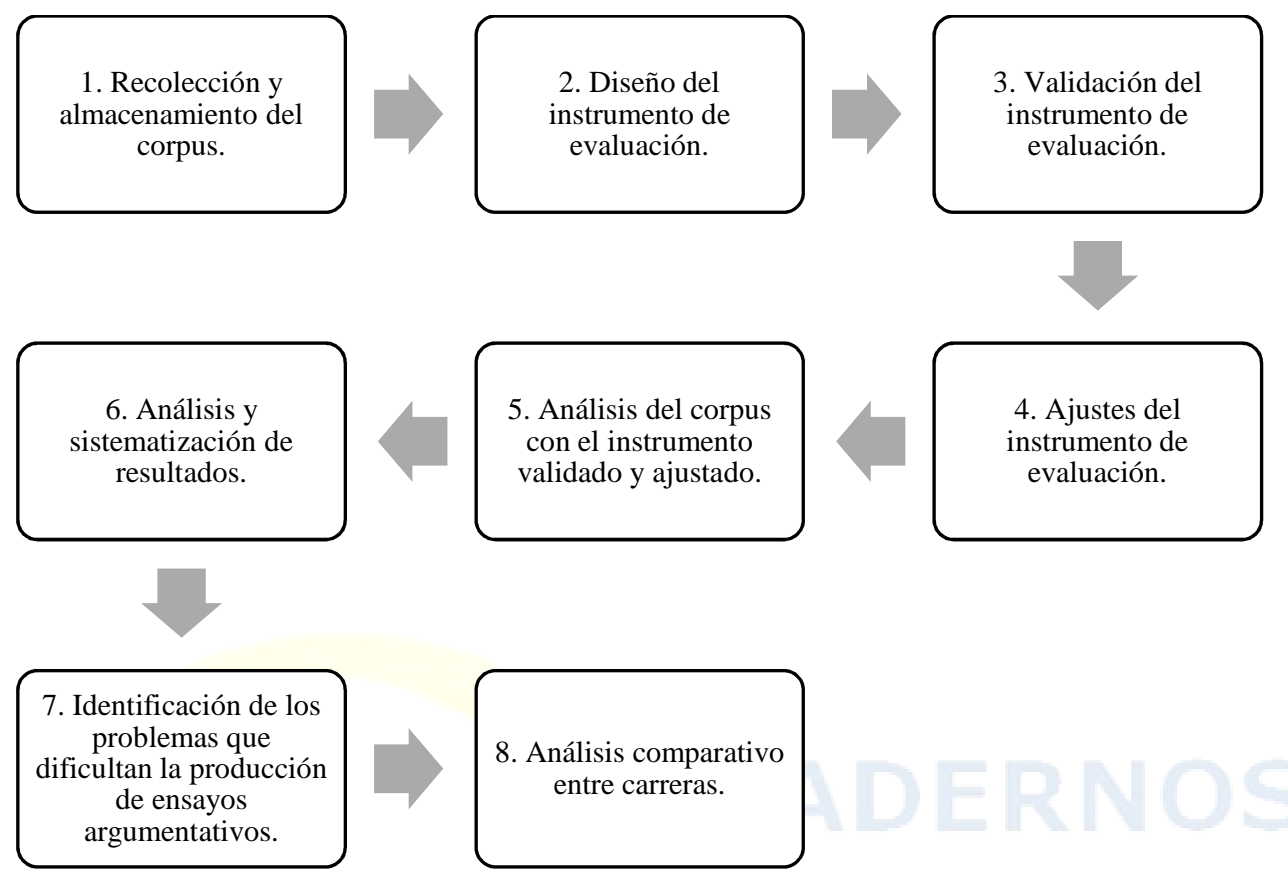

Fuente: Preparado por la autora

\section{ANÁLISIS Y DISCUSIÓN DE RESULTADOS}

En primer lugar, en el Gráfico 1, damos cuenta del porcentaje de logro alcanzado por los estudiantes con sus ensayos. Se trata de un resultado general, a nivel de carrera, que nos permite visualizar el desempeño general de los estudiantes en cada una de las pedagogías consideradas en esta investigación: Pedagogía en Educación General Básica (en adelante, PEGB), Pedagogía en Educación Diferencial (EDI) y Pedagogía en Castellano y Filosofía (CAFILO). 
Gráfico 1- Porcentaje de logro por carrera

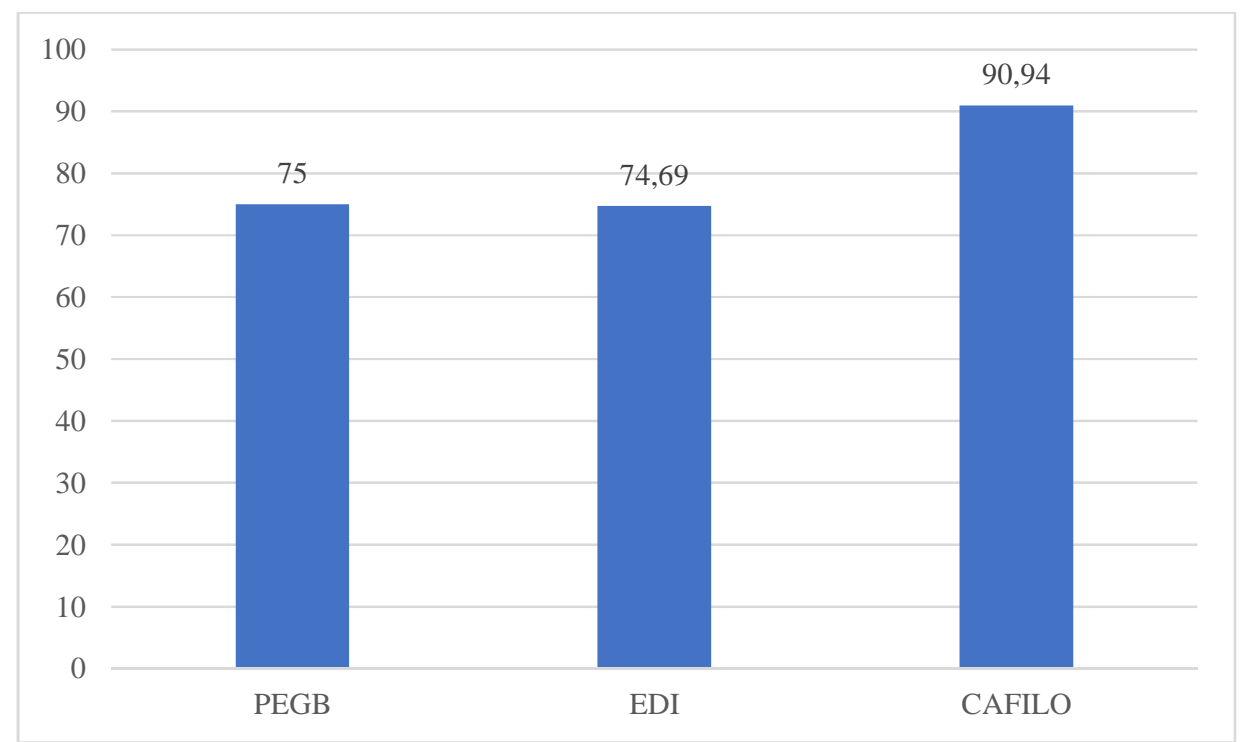

Leyenda: PEGB (Pedagogía en Educación General Básica), EDI (Pedagogía en Educación Diferencial), CAFILO (Pedagogía en Castellano y Filosofía).

Fuente: Preparado por la autora

Como se observa en el Gráfico 1, los estudiantes de PEGB y EDI presentan un porcentaje de logro bastante similar en sus ensayos: $75 \%$ y $74,69 \%$, respectivamente. Mientras que los estudiantes de CAFILO alcanzan un desempeño más alto (90,94\%). En consecuencia, en términos generales, en las tres carreras consideradas se supera el porcentaje mínimo de aprobación que, como hemos señalado, corresponde al $60 \%$. Desde nuestro punto de vista, estos resultados exitosos en las tres carreras de formación de profesores podrían atribuirse al hecho de que los ensayos que hemos analizado son parte de un programa de formación en que los estudiantes reciben semanalmente insumos teóricos y actividades prácticas que pueden aplicar en su trabajo. Además, tienen la posibilidad de solicitar retroalimentación en cualquier momento a la profesora a cargo del curso.

Con respecto al mejor desempeño logrado por parte de los estudiantes de CAFILO, a nuestro juicio, es un resultado que podría deberse a diferentes factores. En primer lugar, debemos recordar que los ensayos analizados fueron entregados 
voluntariamente por los estudiantes. En el caso de CAFILO, curiosamente, quienes accedieron a facilitar sus trabajos con fines investigativos fueron principalmente aquellos estudiantes que obtuvieron muy buenas calificaciones en sus ensayos. De hecho, 3 de los 4 ensayos analizados de esta carrera fueron calificados con la nota máxima, pues alcanzaron un $100 \%$ de logro. En el caso de EDI, si bien todos los ensayos recibidos fueron trabajos aprobados, solo 2 de ellos fueron calificados con la nota máxima. Por el contrario, en el caso de PEGB, el 50\% de los estudiantes que proporcionaron sus ensayos para esta investigación no alcanzaron la nota mínima de aprobación. En este sentido, quizás, podría haber en los estudiantes de CAFILO un menor grado de tolerancia a la frustración, por lo que se atreven a facilitar sus trabajos con fines investigativos solo si han obtenido excelentes resultados. En el caso contrario, los estudiantes de PEGB podrían ver en sus trabajos no logrados una oportunidad para que otras personas aprendan de sus errores, por lo que los facilitan para ser analizados en un contexto investigativo, incluso, si han obtenido malos resultados.

A fin de robustecer los resultados descritos en el párrafo anterior, sería interesante complementar esta investigación con entrevistas para indagar en las razones que tienen los estudiantes para consentir o no que sus trabajos sean utilizados con fines investigativos. En esta línea, sería interesante también indagar en los factores emocionales que influyen tanto en el desempeño en una tarea de escritura como también en el hecho de que un estudiante se atreva o no a entregar su escrito para que sea analizado en una investigación como la que aquí presentamos. Esto cobra mayor sentido si consideramos que se ha reportado que la angustia invade al estudiante al pensar en la opinión que se harán de él los lectores de su texto (ERRÁZURIZ et al., 2015). Este sentimiento de inseguridad y angustia podría aumentar si no solo se leerá su trabajo, sino que, además, será objeto de investigación. Quizás, un constructo que podría entregarnos pistas importantes al respecto es el de percepción de autoeficacia en la escritura académica (GONZÁLEZ, MEZA y CASTELLÓN, 2019; MEZA y GONZÁLEZ, 2020). 
A continuación, en el Gráfico 2, presentamos los porcentajes de logro alcanzados en cada dimensión evaluada en las tres carreras consideradas en esta investigación. 


\section{Gráfico 2 - Porcentaje de logro por dimensión}

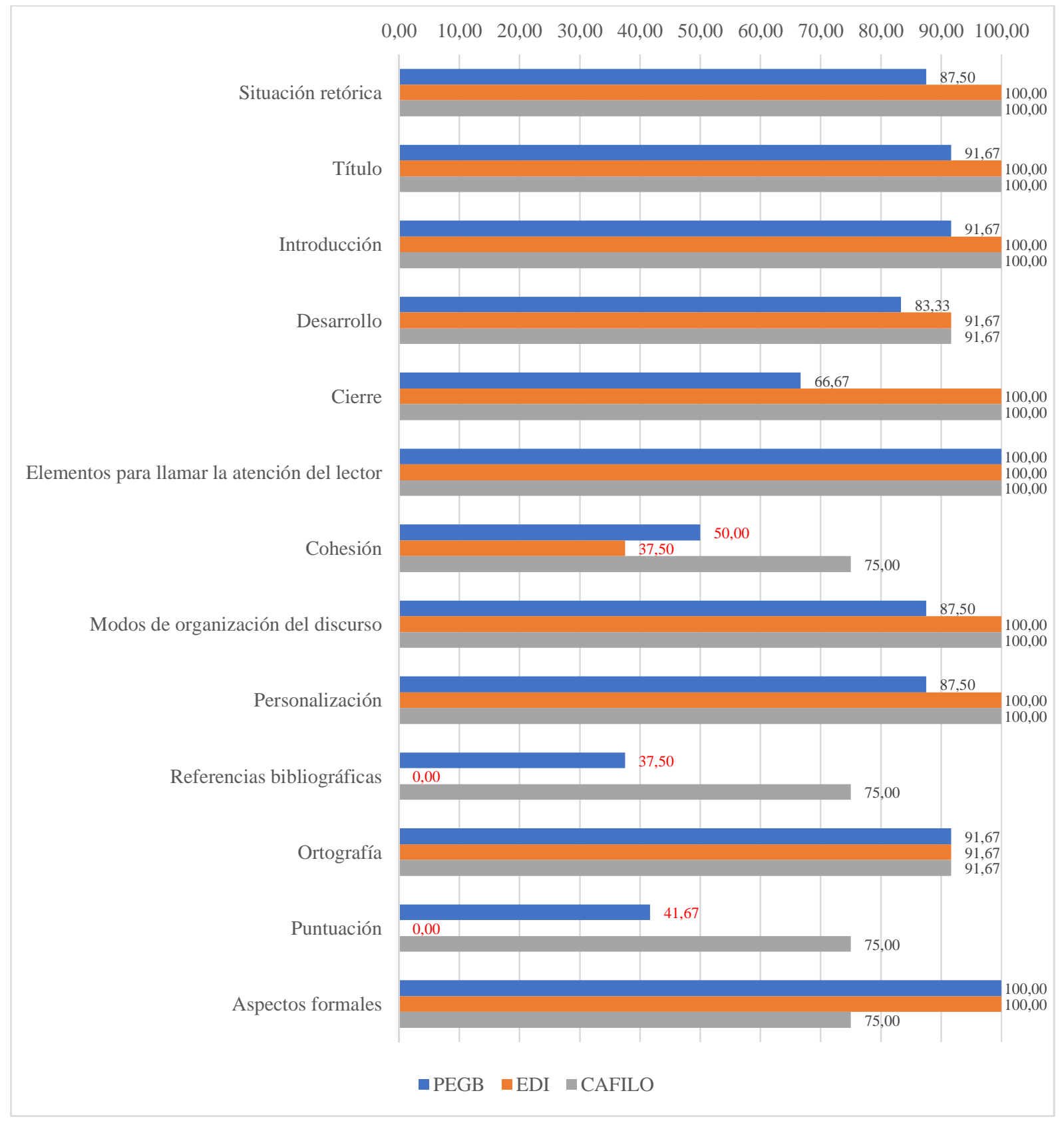

Leyenda: PEGB (Pedagogía en Educación General Básica), EDI (Pedagogía en Educación Diferencial), CAFILO (Pedagogía en Castellano y Filosofía).

Fuente: Preparado por la autora 
Como se observa en el Gráfico 2, existe una dimensión que alcanza el 100\% de logro en las tres carreras de formación de profesores incluidas en esta investigación, a saber, la inclusión de elementos que permiten captar la atención del lector. Esto indica que todos los textos analizados en esta investigación evidencian un dominio excelente de la inclusión de citas, anécdotas, analogías o cualquier otro elemento que permita mantener la atención del lector. Un ejemplo de ello es el uso de la siguiente cita en un ensayo sobre eutanasia: «Séneca (2020: en línea) expresa que "la muerte es un castigo para algunos, para otros un regalo, y para muchos un favor"» [CAFILO_3]. Otro ejemplo es el uso de preguntas retóricas para introducir el tema de la ley de acoso escolar en Chile: “¿Qué tan eficaz es la ley actual de acoso escolar que resguarda a los chilenos?, ¿la ley existente considera a todos los establecimientos educativos chilenos dentro del consejo escolar?, ¿de qué manera se han visto afectadas las víctimas de acoso?" [PEGB_1].

Otro elemento que alcanza un altísimo nivel de logro en las tres carreras es la dimensión Título, dirigida a evaluar que el título del ensayo sea representativo del tópico tratado y, además, atractivo para el lector. En este caso, tanto los trabajos de EDI como de CAFILO alcanzan un 100\% de logro, mientras que en PEGB obtienen, en promedio, un $91,67 \%$ de logro. Un ejemplo de título que no obtuvo el puntaje máximo es "La influencia del internet en la educación" [PEGB_4]. En este caso, como puede observarse, si bien el título es descriptivo, no resulta atractivo; incluso, podría tratarse de una frase que titule un trabajo expositivo.

La Introducción es otra dimensión que presenta un alto nivel de logro en las tres carreras estudiadas. Nuevamente, tanto los trabajos de EDI como de CAFILO alcanzan un $100 \%$ de logro, mientras que en PEGB obtienen, en promedio, un $91,67 \%$ de logro. Este resultado indica que la gran mayoría de los ensayos analizados logran dar cuenta correctamente de la introducción de su trabajo y, además, incluyen todas las movidas retóricas propias de esta sección en el género en cuestión. Este buen desempeño de los estudiantes en la introducción de su ensayo podría deberse, primero, a que tempranamente, en la planificación de su 
escritura, ya han redactado al menos tres de las movidas nucleares de la introducción, por lo que varios de esos elementos podrían encontrarse revisados y corregidos incluso antes de comenzar la redacción misma del ensayo. Además, dado que la introducción es, en general, la primera sección que escriben del ensayo, en consecuencia, es también una de las que más retroalimentación recibe.

Ahora bien, en cuanto a las dimensiones no logradas, vale decir, las que no alcanzan el 60\% de logro, son las que se han marcado en rojo en el Gráfico 2. En primer lugar, el uso adecuado de mecanismos de cohesión es una dimensión en la que tanto los estudiantes de PEGB como de EDI obtienen una puntuación deficiente (50\% y $37,5 \%$, respectivamente). Ello da cuenta de que los estudiantes de estas carreras tuvieron serios problemas para utilizar adecuadamente los diversos mecanismos de cohesión para organizar su texto y unir sus ideas (marcadores discursivos, correferencia, repeticiones, etc.). Esto indica que se debe realizar un trabajo importante de reforzamiento o bien la búsqueda de nuevas estrategias metodológicas para consolidar el dominio de los mecanismos de cohesión por parte de los estudiantes. Ello resulta fundamental si consideramos que el uso de dichos mecanismos no es exclusivo del ensayo argumentativo, sino que trasciende a la escritura académica e, incluso, a la producción de textos en general.

Las dificultades en el ámbito de la cohesión evidencian que los estudiantes que ingresan a carreras de pedagogía no han logrado dominar aspectos generales de la producción de textos. En este sentido, a nuestro juicio, sería errónea la consideración tácita de que, en contextos universitarios, los alumnos desde su ingreso ya han sido alfabetizados y, por tanto, cuentan con las herramientas básicas para producir textos, pues escribir, y leer, desde hace ya varios años y niveles escolares (VILLASEÑOR, 2019).

Los resultados obtenidos por los estudiantes en la dimensión puntuación vendrían a corroborar la idea expuesta en el párrafo anterior. Así, los ensayos de los estudiantes de PEGB y EDI alcanzaron una puntuación bastante deficiente en esta dimensión que valora el uso correcto de la puntuación. De hecho, los ensayos 
de EDI obtuvieron un 0\% de logro en la dimensión en cuestión, lo que evidencia graves deficiencias en los estudiantes, a pesar de que recibieron clases teóricas y desarrollaron actividades prácticas para trabajar este aspecto. Este resultado es coincidente con otras investigaciones que han indagado en los problemas de escritura en estudiantes de pregrado. Así, por ejemplo, Meza, Castellón y Gladic (2021) identificaron que la puntuación es uno de los aspectos que reviste mayor dificultad al momento de producir un texto para estudiantes de Medicina y Derecho de una universidad chilena. De hecho, los textos de Medicina analizados en esa investigación también reportaron un $0 \%$ de logro en el ítem puntuación por parte de los estudiantes de Medicina. Esto, sumado a los resultados de esta investigación, sugeriría que el desconocimiento de las normas de puntuación podría ser un problema transversal a los estudiantes universitarios en Chile. En este sentido, desde nuestro punto de vista, valdría la pena revisar el trabajo que se está realizando durante la educación primaria y secundaria para preparar a los estudiantes en producción de textos en general y en puntuación en particular.

La última dimensión que reviste mayor complejidad para los estudiantes de las carreras de pedagogía estudiadas es la denominada Referencias bibliográficas. Ella permite evaluar que todas las citas y referencias incluidas en los ensayos de los estudiantes se ajusten al formato de las normas APA (American Psychological Associaton) versión 7. En esta dimensión, advertimos que los ensayos de EDI no logran superar el 0\% de logro, mientras que los de PEGB alcanzan un 37,5\%; los estudiantes de CAFILO, por su parte, obtienen un $75 \%$ de logro. Este resultado evidencia el grave problema que poseen los estudiantes para integrar otras fuentes en su ensayo. La puntuación deficiente en esta dimensión es coincidente con los resultados reportados por Errázuriz et al. (2015), quienes estudiaron el género ensayo producido por estudiantes de Pedagogía Básica de una universidad chilena. Las autoras atribuyen este problema a la presión que produce la situación de escribir, razón por la cual muchos estudiantes olvidan el modo de citar correctamente. A ello agregan que una cierta cantidad de estudiantes, al entrar a la 
universidad, desconocen las normas para citar textos (ERRÁZURIZ et al., 2015), por lo que se trataría de un conocimiento que deben aprender desde cero.

Por otro lado, Ilama la atención el caso de la dimensión "Aspectos formales", que valora que el trabajo cumple con las especificaciones de formato indicadas (tipo y tamaño de fuente, interlineado, etc.). Los resultados obtenidos son atrayentes, pues EDI y PEGB, que son las carreras que, en general, presentaron mayores problemas, alcanzan un $100 \%$ de logro. Mientras que CAFILO, que es la carrera con mejores resultados, logra solo un 75\%. En este sentido, aparentemente, si bien los ensayos de CAFILO logran ensayos argumentativos más exitosos, parecen poner menos atención en cuestiones de formato.

Finalmente, nos parece importante relevar que, en términos generales, los ensayos evaluados en esta investigación han obtenido mejores resultados que los reportados por otras investigaciones similares recientes como, por ejemplo, la de Meza, Castellón y Gladic (2021), donde se identifican los problemas frecuentes en textos profesionales con fines académicos producidos por estudiantes de Derecho y Medicina. Probablemente, esto se debe a que los estudiantes participantes en esta investigación tienen un curso para adquirir las competencias necesarias para desarrollar el ensayo y, además, contaron con el acompañamiento de la profesora a cargo de los cursos durante todo el proceso de escritura del ensayo.

\section{CONCLUSIONES}

En esta investigación propusimos como objetivos a) identificar los problemas que dificultan la producción de ensayos argumentativos por parte de estudiantes de primer año de tres carreras de pedagogía de una universidad chilena; b) determinar la variación disciplinar de los problemas identificados en la producción de ensayos argumentativos por parte de estudiantes de primer año de tres carreras de pedagogía de una universidad chilena. Los resultados centrales que nos han entregado su cumplimiento son, primero, la identificación de las dimensiones 
específicas que causan mayores dificultades a los estudiantes mencionados al momento de producir un ensayo argumentativo. Así, podemos afirmar que para los estudiantes de PEGB y EDI las dimensiones más problemáticas son el uso de mecanismos de cohesión, la integración de referencias bibliográficas de acuerdo con los requerimientos de las normas APA y el uso adecuado de la puntuación según las normas de la lengua española. En el caso de CAFILO, si bien no identificamos dimensiones bajo el porcentaje de logro (60\%), igualmente, tienen un resultado menos exitoso en las mismas dimensiones ya mencionadas. Por otro lado, la consecución de los objetivos nos ha permitido afirmar, además, que son los estudiantes de CAFILO, quienes, en general, tienen menos problemas en la producción de ensayos argumentativos.

Por supuesto, dadas las características de la muestra y el diseño de la investigación desarrollada, los resultados que hemos presentado no son generalizables a grupos más amplios. Además, como ya hemos señalado, podría existir un sesgo en los datos en el sentido de que solo hemos analizado aquellos ensayos que los estudiantes han facilitado voluntariamente.

Creemos que la investigación presentada es un aporte para la didáctica de la escritura en la formación de profesores, pues ofrecemos las dimensiones específicas del ensayo que revisten mayor complejidad para los estudiantes. En consecuencia, gracias a la identificación de esas dimensiones más complejas, contamos con datos para saber qué elementos del ensayo deben trabajarse con mayor dedicación o bien con una estrategia diferente. Creemos que, en la universidad contemporánea, es fundamental contar con este tipo información para que los estudiantes sean capaces producir conocimiento acorde con los requerimientos de su comunidad discursiva.

En cuanto a las proyecciones de esta investigación, nos interesa, primero, aumentar el tamaño del corpus. En segundo lugar, nos parece atractivo tener la posibilidad de complementar los resultados aquí obtenidos con entrevistas, grupos focales o aplicación de cuestionarios para profundizar en la interpretación y 
discusión de los resultados. Esto nos permitiría contar con una visión más integral de la producción de textos académicos en carreras de formación de profesores. Finalmente, esperamos poder contrastar los resultados obtenidos en esta investigación con los resultados obtenidos por los mismos estudiantes en su evaluación diagnóstica, desarrollada antes de tomar el curso de comunicación oral y escrita.

\section{REFERENCIAS}

AL FADDA, Hind. Difficulties in academic writing: From the perspective of King Saud university postgraduate students. English Language Teaching, v. 5, n. 3, 123-130, 2012.

ARECHABALA, María Cecilia; CATONI, María Isabel; ÁVILA, Natalia; RIQUELME, Gisselle; AEDO, Vivian. Géneros discursivos y errores más frecuentes en los informes académicos de estudiantes de enfermería. Investigación y Educación en Enfermería, v. 29, n. 3, 400-406, 2011.

CASTRO, María Cristina; HERNÁNDEZ, Laura Aurora; SÁNCHEZ, Martín. EI ensayo como género académico. Una aproximación a las prácticas de escritura en la universidad pública mexicana. En: PARODI, Giovanni (ed.). Alfabetización académica y profesional en el siglo XXI: Leer y escribir desde las disciplinas. Santiago: Ariel, 2010. p. 49-70.

CRESWELL, John; CRESWELL, J. David. Research Design. Qualitative, Quantitative, and Mixed Methods Approaches. Thousand Oaks: Sage, 2018.

ERRÁZURIZ, María Constanza. Análisis del uso de los marcadores discursivos en argumentaciones escritas por estudiantes universitarios. Perfiles Educativos, v. 34, n. 136, p. 98-117, 2012.

ERRÁZURIZ, María Constanza; ARRIAGADA, Lucía; CONTRERAS, Maritza; LÓPEZ, Carla. Diagnóstico de la escritura de un ensayo de alumnos novatos de Pedagogía en el campus Villarrica UC, Chile. Perfiles Educativos, v. 37, n. 150, p. 76-90, 2015.

ERRÁZURIZ, María Constanza. Desempeño escrito de estudiantes de programas de formación inicial docente: ¿Cómo es la calidad del proceso de escritura de sus ensayos? Lengua y Habla, n. 23, p. 224-242, 2019. 
GONZÁlEZ, Mauricio; MEZA, Paulina; CASTELLÓN, Melissa. Medición de la Autoeficacia para la Escritura Académica: una revisión teórico-bibliográfica. Formación Universitaria, v. 12, n. 6, p. 191-204, 2019.

HERNÁNDEZ, Roberto; FERNÁNDEZ, Carlos; BAPTISTA, Pilar. Metodología de la investigación. México D.F.: McGraw-Hill Interamericana, 2014.

HERMOSILLO, Perla; VERdíN, Paulo. Perspectivas de la escritura académica en la universidad: causas, problemáticas y propuestas. Cuaderno de Pedagogía Universitaria, v. 16, n. 31, p. 41-57, 2019.

HUANG, Yu; JUN ZHANG, Lawrence. Does a process-genre approach help improve students' argumentative writing in English as a foreign language? Findings from an intervention study. Reading \& Writing Quarterly, v. 36, n. 4, p. 339-364, 2020.

IBÁÑEZ, Romualdo. El texto disciplinar y el acceso al conocimiento desde el análisis del género: ¿Regulación del conocimiento o persuasión? En: PARODI, Giovanni. (ed.). Géneros académicos y géneros profesionales: accesos discursivos para saber y hacer. Valparaíso: Ediciones Universitarias de Valparaíso, 2008.

JAWORSKA, Sylvia; KRUMMES, Cedric; ENSSLIN, Astrid. Formulaic sequences in native and non-native argumentative writing in German. International Journal of Corpus Linguistics, v. 20, n. 4, p. 500-525, 2015.

KANOKSILAPATHAM, Budsaba. Rhetorical moves in biochemistry research articles. En: BIBER, Douglas; CONNOR, Ulla; UPTON, Thomas (eds.). Discourse on the move. Ámsterdam: Benjamins, 2007.

KHUWAILEH, Abdullah; SHOUMALI, Ali. Writing errors: A study of the writing ability of Arab learners of academic English and Arabic at university. Language Culture and Curriculum, v. 13, n. 2, p. 174-183, 2000.

MARTÍNEZ, Juan. El género Tesis Doctoral de Historia y Física: descripción y variación retórico-funcional. En Leer y escribir en contextos académicos y profesionales: géneros, corpus y métodos. En: PARODI, Giovanni; BURDILES, Gina (eds.). Leer y escribir en contextos académicos y profesionales. Géneros, corpus y métodos. Santiago: Ariel, 2015.

MEZA, Paulina; DA CUNHA, Iria. Comunicación del conocimiento propio y relaciones discursivas en el género tesis. Sintagma. Revista de Lingüística, v. 31 , p. 103-130, 2019. 
MEZA, Paulina; GONZÁLEZ, Mauricio. Construction and validation of the selfefficacy scale for disciplinary academic writing. Cogent Education, v. 7, n. 1, 2020.

MEZA, Paulina; CASTELLÓN, Melissa; GLADIC, Jadranka. Problemas de escritura en la producción de textos de estudiantes de Derecho y Medicina. DELTA: Documentação de Estudos em Lingüística Teórica e Aplicada, v. 37, p. 1-29, 2021.

NGUYEN, Loan Thi Thuy. A case study of teacher feedback on Thai university students' essay writing. GEMA Online ${ }^{\circledR}$ Journal of Language Studies, v. 19, n. 2, p. 121-138, 2019.

OLAVE-ARIAS, Giohanny; CISNEROS-ESTUPIÑÁN, Mireya; ROJAS-GARCÍA, llene. Deserción universitaria y alfabetización académica. Educación y Educadores, v. 16, n. 3, p. 455-471, 2013.

OYARZÚN, Romina; VALDÉZ, Gabriel. Propuesta didáctica para el desarrollo de habilidades comunicativas en el contexto universitario: un acercamiento a la cultura discursiva en la educación superior. Aularia: Revista Digital de Comunicación, v. 8, n. 1, p. 1-8, 2019.

PARKINSON, Jean; MUSGRAVE, Jill. Development of noun phrase complexity in the writing of English for Academic Purposes students. Journal of English for Academic Purposes, v. 14, p. 48-59, 2014.

PINETEH, Ernest. A. The academic writing challenges of undergraduate students: A South African case study. International Journal of Higher Education, v. 3, n. 1, p. 12-22, 2014.

SHIN, Yu Kyoung. Do native writers always have a head start over nonnative writers? The use of lexical bundles in college students' essays. Journal of English for Academic Purposes, v. 40, p. 1-14, 2019.

URIBE, Olga; CARRILLO, Stefany. Relación entre la lecto-escritura, el desempeño académico y la deserción estudiantil. Entramado, v. 10, n. 2, p. 272-285, 2014.

VILLASEÑOR, Victoria. Hacia una didáctica de la escritura académica en la universidad. Reencuentro. Análisis de problemas universitarios, n. 66, p. 91101, 2019.

WINGATE, Ursula. 'Argument!' helping students understand what essay writing is about. Journal of English for Academic Purposes, v. 11, n. 2, p. 145-154, 2012. 